\title{
Search for Flavor Changing Neutral Current in Top Production and Decays
}

\section{Tae Jeong Kim on behalf of the CMS collaboration}

Hanyang University, Seoul, South Korea

E-mail: taekim@hanyang.ac.kr

Searches for flavor changing neutral currents in top production and decay using data collected by the Compact Muon Solenoid (CMS) experiment at $\sqrt{s}=7$ and $8 \mathrm{TeV}$ are presented, corresponding to an integrated luminosity of around $5 \mathrm{fb}^{-1}$ and $20 \mathrm{fb}^{-1}$. FCNC searches are conducted to probe $t q Z, t q \gamma, t q H$, and $t g q$ interactions in various channels. By the time of the $38^{t h}$ ICHEP conference in 2016, the upper limits on $\mathscr{B}(t \rightarrow u \gamma)<0.013 \%, \mathscr{B}(t \rightarrow u g)<0.036 \%, \mathscr{B}(t \rightarrow u Z)<0.05 \%$ and $\mathscr{B}(t \rightarrow u H)<0.42 \%$ at the $95 \%$ confidence level had been obtained by the CMS collaboration. 


\section{Introduction}

In the standard model (SM), the Flavor Changing Neutral Current (FCNC) can occur only at the level of quantum loop correction with the branching ratio of less than $10^{-13}$. However, a wide variety of models beyond the SM shows a strong dependence in the measurable FCNC quantities, e.g. the branching ratio of top decaying to a charm quark and a Higgs boson in 2HDM is around $10^{-4}$. Therefore, the study of FCNC is one of the most interesting research topic in top quark physics. The Compact Muon Solenoid (CMS) experiment [1] at the LHC has accumulated data corresponding to an integrated luminosity of around $5 \mathrm{fb}^{-1}$ at $\sqrt{s}=7 \mathrm{TeV}$ in 2011 and $20 \mathrm{fb}^{-1}$ at $\sqrt{s}=8 \mathrm{TeV}$ in 2012. Searches for FCNC in top production and decay using data collected by the CMS experiment at the time of the ICHEP 2016 conference are presented. FCNC searches are conducted to probe $t q Z, t q \gamma, t q H$, and $t g q$ interactions in various channels.

\section{Search for $t q Z$ coupling in top decay}

Top-quark pair events where one of top decays to a $\mathrm{Z}$ boson and a up-type quark have been analyzed searching for the coupling of $t q Z$ with data corresponding to an integrated luminosity of $4.9 \mathrm{fb}^{-1}$ collected at $7 \mathrm{TeV}$ and $19.7 \mathrm{fb}^{-1}$ at $8 \mathrm{TeV}$ [2]. Events are selected with three leptons $(e, \mu)$ : two leptons from a $\mathrm{Z}$ boson and one lepton from a $\mathrm{W}$ boson in top decays. The distribution of reconstructed top quark mass in the SM decay (left) and FCNC decay (right) are shown in Fig. 1 which show a good agreement between data and the SM background prediction. An observed limit on the branching ratio of $\mathscr{B}(t \rightarrow Z q)<0.5 \%$ is obtained.
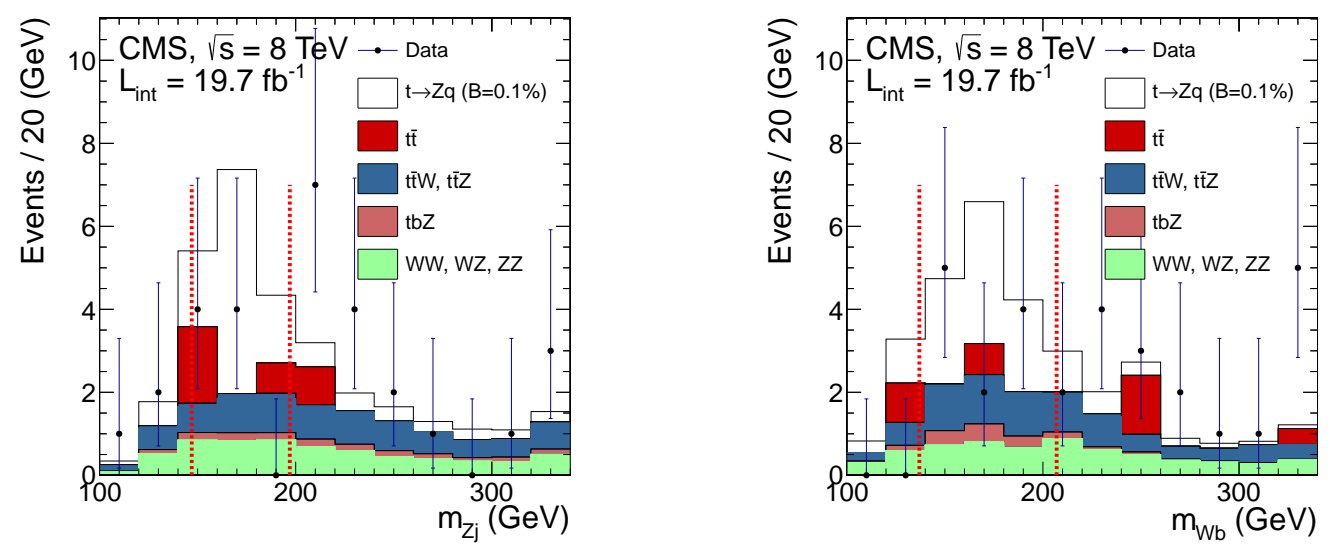

Figure 1: The distributions of reconstructed top quark mass in the SM decay (left) and FCNC decay (right) are shown.

\section{Search for $t q H$ coupling in top decay}

The search for the rare flavor-changing decay of the top quark to a Higgs boson and a charm or up quark $[3,4,5]$ have also been performed. Top-quark pair events in the hadronic and leptonic channel, where the Higgs boson decays two photons are used in this search. Figure 2 shows the diphoton invariant mass distributions for hadronic channel (left) and leptonic (right) channel. The 
$\gamma \gamma$ resonant from the SM Higgs is taken into account in the background modeling. No excess over the data is found in both analysis, being the sensitivity driven by the hadronic channel. The result from the final state of diphoton search analysis combining two channels is $\mathscr{B}(t \rightarrow c H)<$ $0.47 \%$ and is $0.42 \%$ for $\mathscr{B}(t \rightarrow u H)$. The main uncertainties are from the photon identification and non-resonant background estimation.
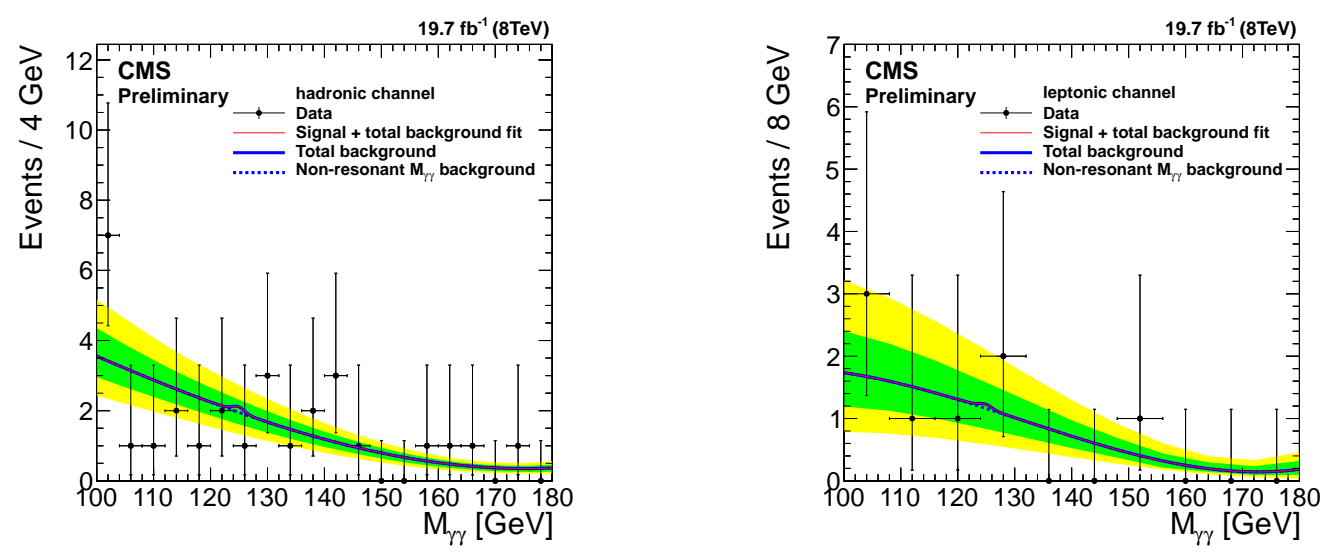

Figure 2: Diphoton invariant mass distributions and fit result for the hadronic channel (left) and the leptonic channel (right). The blue solid line indicates the total background including the resonant from the Higgs boson and the non-resonant diphoton background.

\section{Search for $t q \gamma$ coupling in single top production}

The anomalous $t q \gamma$ coupling has been searched with events in association with a photon using the full data collected at $8 \mathrm{TeV}$ in 2012, corresponding to $19.1 \mathrm{fb}^{-1}$ [6]. The corresponding Feynman diagram is shown in Fig 3 (left). In order to reduce the QCD multijet background, the top quark in the lepton decay mode is considered. Therefore, one isolated muon, one isolated photon and one $b$-tagged jet are required to select the final signature. A Boosted Decision Tree (BDT) is used to separate the signal signature from the background contributions. There is no excess observed. Photon energy scale and the estimate of $W \gamma+$ jets process are the main uncertainty sources in this analysis. The upper limits on the branching ratios of $t u \gamma$ and $t c \gamma$ is shown in Fig 3 (right), being the case of coupling with c-quark and u-quark indicated by red vertical line. The observed limits on the branching ratios of $\mathscr{B}(t \rightarrow u \gamma)<0.013 \%$ and $\mathscr{B}(t \rightarrow c \gamma)<0.17 \%$ are obtained. This result is the first measurement of the limit on these couplings at the LHC and provide the most stringent bounds on the anomalous FCNC $t q \gamma$ coupling to date.

\section{Search for $t q g$ coupling in single top production}

The anomalous couplings of $t \mathrm{cg}$ and $t u g$ were searched in the $t$-channel single top-quark production using data collected at $7 \mathrm{TeV}$ corresponding to an integrated luminosity of $5 \mathrm{fb}^{-1}$ [7]. The 

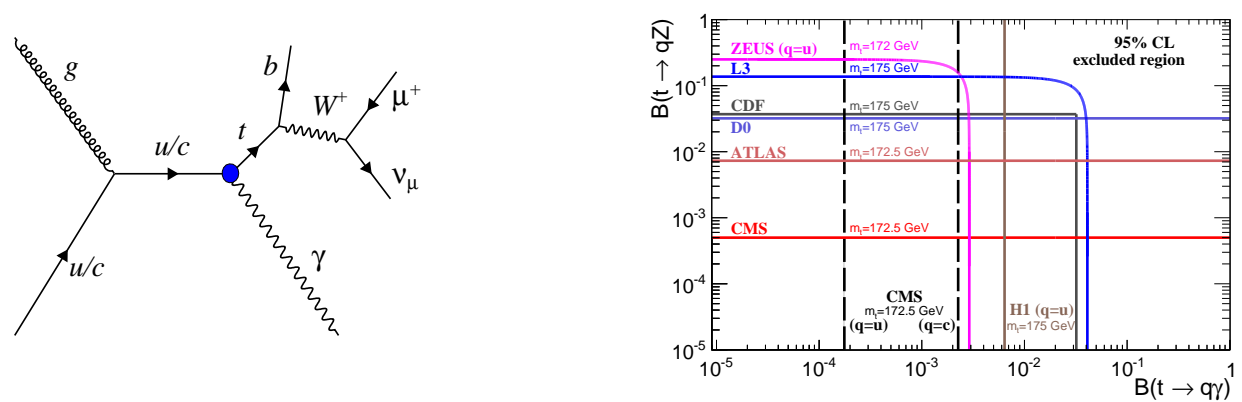

Figure 3: (Left) Lowest-order Feynman diagram for single top quark production in association with a photon via a FCNC. (Right) The measured 95\% CL upper limits on $\mathscr{B}(t \rightarrow q Z)$ versus $\mathscr{B}(t \rightarrow q \gamma)$. The two vertical dashed lines show the results of this analysis for the $t \rightarrow q \gamma$ coupling.

final signature of the anomalous FCNC coupling is the same as the SM single top quark production processes with one isolated muon, 2 or 3 jets and one $b$-tagged jet. However, the signal has different kinematic distributions and is separated from the backgrounds using a Bayesian Neural Network (BNN) based on the distinctive kinematical properties. Data and the SM predictions agree well within the uncertainties. In this analysis, one of the main sources of uncertainty is the estimate of the $\mathrm{W}+$ jets contribution to the signal region. Taking into account the systematic uncertainties, the limit on the couplings of $t c g$ and $t u g$ are calculated at the $95 \%$ confidence level. The observed upper limits of $\mathscr{B}(t \rightarrow u g)<0.036 \%$ and $\mathscr{B}(t \rightarrow c g)<0.34 \%$ are obtained.

\section{Conclusions}

The CMS collaboration has performed several FCNC searches in both top-quark pair and a single top-quark production and decay, with data collected at 7 and $8 \mathrm{TeV}$. No sign of FCNC contribution in the top sector has been found so far. Limits on the branching ratios $\mathscr{B}(t \rightarrow u \gamma)<0.013 \%$, $\mathscr{B}(t \rightarrow u g)<0.036 \%, \mathscr{B}(t \rightarrow Z q)<0.05 \%$ and $\mathscr{B}(t \rightarrow u H)<0.42 \%$ at $95 \%$ confidence level are obtained. More results from Run 1 are expected to come soon. Exciting time are ahead of us with data to be collected at $13 \mathrm{TeV}$, which will allow the LHC experiments to probe further the contributions from rare FCNC processes in the top sector. After the $38^{\text {th }}$ ICHEP conference, more results [8] on the FCNC became available.

\section{References}

[1] CMS Collaboration, "The CMS experiment at the CERN LHC", JINST 3:S08004,2008.

[2] CMS Collaboration, "Search for Flavor-Changing Neutral Currents in Top-Quark Decays $t \rightarrow Z q$ in $p p$ Collisions at $\sqrt{s}=8 \mathrm{TeV}$ ", Phys. Rev. Lett., 112 (2014) 171802.

[3] CMS Collaboration, "Search for top quark decays $t \rightarrow q H$ with $H \rightarrow \gamma \gamma$ in pp collisions at $\sqrt{s}=8$ TeV $p p^{\prime}$, PAS TOP-14-019.

[4] CMS Collaboration, "Search for top quark decays via Higgs-boson-mediated flavor changing", PAS TOP-13-017, arXiv:1610.04857.

[5] CMS Collaboration, "Search for the flavor-changing neutral current decay $t \rightarrow q H$ where the Higgs decays to $b \bar{b}$ pairs at $\sqrt{s}=8 \mathrm{TeV}$ ", PAS TOP-14-020. 
[6] CMS Collaboration, "Search for anomalous single top quark production in association with a photon in pp collisions at $\sqrt{s}=8 \mathrm{TeV} "$, JHEP 04 (2016) 035.

[7] CMS Collaboration, "Search for anomalous Wtb couplings and top FCNC in t-channel single-top-quark events", PAS TOP-14-007.

[8] https://twiki.cern.ch/twiki/bin/view/CMSPublic/PhysicsResultsTOP. 